\title{
Marlowe : de quelques problèmes d'interprétation
}

Jean Jacquot

Marie-Thérèse Jones-Davies (ed.)

\section{(2) OpenEdition}

Electronic version

URL: http://journals.openedition.org/shakespeare/451

DOI: 10.4000/shakespeare.451

ISSN: 2271-6424

Publisher

Société Française Shakespeare

Printed version

Date of publication: 1 November 1981

Number of pages: 201-238

ISBN: 2-86433-016-4

\section{Electronic reference}

Jean Jacquot, « Marlowe : de quelques problèmes d'interprétation », Actes des congrès de la Société française Shakespeare [Online], 3 | 1981, Online since 01 November 2007, connection on 22 January 2020. URL : http://journals.openedition.org/shakespeare/451 ; DOI : 10.4000/shakespeare.451 


\title{
THEATRE ET IDEOLOGIES :
} Marlowe, Shakespeare

\author{
DIRECTEUR DE LA PUBLICATION \\ M.T. Jones - Davies
}

JEAN TOUZOT Libraire - Editeur

38 , rue Saint-Sulpice 75278 PARIS CEDEX 061982 
1982 Jean Touzot Libiaire-Editeur, Paris.

Reproduction et traduction mzme partielle interdite.

Tous droits réservts potir lous pays

y compris i'U,R.S.S. et les pays scandinaves

ISBN 2-86433-016-4 


\title{
AVANT-PROPOS
}

L'opposition soulignée par Horace entre éthique et esthétique Aut prodesse volunt aut delectare poetae - que les Elisabéthains aiment à évoquer, pourrait être le lien qui rattache les unes aux autres les communications, rencontres et table ronde de notre troisième congrès (1981) sur le thème : "Théátre et Idéologies: Marlowe, Shakespeare.» Toutefois, le résultat de nos échanges montre un dépassement possible de l'alternative éthique / esthétique, puisqu'il met en valeur l'insertion d'idéologies susceptibles de transformer la fonction même de la littérature.

Les pièces analysées dans le contexte de leur temps révèlent de multiples aspects de la vision du monde et de la place que l'homme $y$ occupe, tels que les projette le théâtre anglais au tournant du XVIe et au début du XVIIe siècle.

Une exploration du langage et de la pensée des deux grands dramaturges aboutit à un commentaire sur les courants philosophiques ou religieux, sur les idées parfois contradictoires et sur la curiosité intellectuelle jamais assouvie qui se font jour à travers la rhétorique de la scène et le jeu dramatique, du Faust de Marlowe à La Tempéte de Shakespeare.

\author{
M.T. jones-Davies
}

Présidente de la Société Française Shakespeare 
SOCIETE FRANCAISE SHAKESPEARE Actes de Congrès.1981

\section{THEATRE ET IDEOLOGIES : Marlowe, Shakespeare}

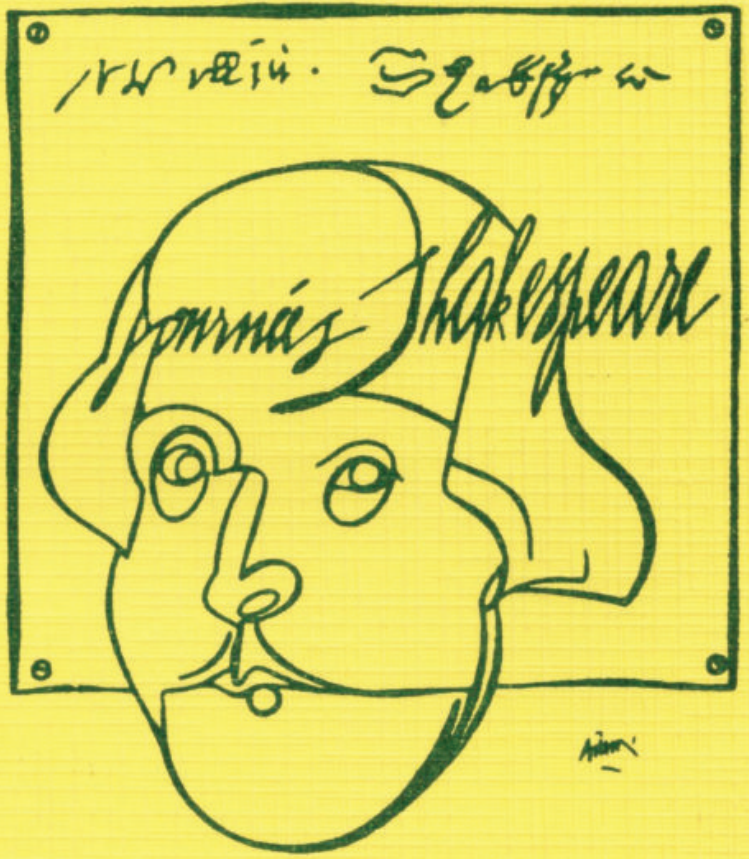

DIRECTEUR DE LA PUBLICATION M.T. Jones - Davies

JEAN TOUZOT Libraire - Editeur 38 , rue Saint-Sulpice 75278 PARIS CEDEX 061982 
Illustration de la couverture : affiche de Valerio Adami, spécialement conçue pour les Journées Shakespeare 1979 au Centre Georges Pompidou 


\section{TABLE DES MATIERES}

M.T. JONES-DAVIES Avant-propos

Théâtre et Idéologies: Marlowe, Shakespeare

S. TRUCHET

The Alchemy of Beauty: aesthetic principles in I Tamburlaine and their relationship to the hermetic ideology.

D. PRUDHOMME

L'idéologie du Juif de Malte et du Marchand de Venise.

D. GOY-BLANQUET

De Hall à Shakespeare : quelques glissements idéologiques opérés par la dramatisation dans Henry $I V$.

M.T. JONES-DAVIES Le Monıde du «si»: Idéologies Incertaines dans Comme il vous Plaira.

G. VENET

Temps et Idéologie : Marlowe, Shakespeare.

J. RICHER

Les Sept Caractères et les Quatre Tempéraments dans Jules César de Shakespeare.

F. LAROQUE

En marge de l'Idéologie : Antimasque et Grotesque dans le Dr Faustus et La Tempête.

A. LECERCLE-SWEET Conscience et Méconnaissance dans Edward The Second de Marlowe.

M.A. CONEJERO Shakespeare's Aesthe tic Scheme. (Extrait).

P.STEWART

Shylock, Shakespeare's Alien.

(Royal Shakespeare Company)

J.RUSSELL BROWN The Romantics' Shakespe are.

R. GILL

The Christian Ideology of Dr Faustus.

J. JACQUOT

Marlowe : de quelques problèmes d'interprétation. 
Rencontres entre Universitaires et Gens de Théâtre

Vendredi 4 décembre 1981
- Stuart Seide :Présentation du

Songe d'une nuit d'Eté. Mise en scène pour le Théâtre de Chaillot janvier 1982

- A propos d'Edouard (II) de

Bernard Turle

Bernard Turle : Métamorphoses de l'auteur et de l'écriture dramatiques. Intervention de Colin Harris (metteur en scène) 


\section{MARLOWE : DE QUELQUES PROBLEMES D'INTERPRETATION}

En schématisant quelque peu on pourrait dire que dans les études marloviennes ont prévalu successsivement deux tendances. Beaucoup ont vi en lui un poète aux hautes aspirations, exaltant l'«individualisme» de la Renaissance, une figure fascinante et provocante de rebelle. Puis d'autres, par réaction, ont estimé que les documents sur sa réputation avaient trop influencé l'interprétation de ses oeuvres, et se sont attachés à démontrer la conformité de ses tragédies avec l'orthodoxie religieuse, voire à faire de lui un moraliste chrétien. Cette réaction a eu des effets positifs car elle a contribué à mettre en évidence la culture biblique et théologique dont témoignent ses pièces et surtout l'utilisation de ces données comme éléments de structure et source d'effets dramatiques. Mais s'il y a beaucoup à apprendre dans les travaux où domine l'une ou l'autre conception, leurs auteurs n'ont pas toujours su résister à la tentation de projeter l'homme Marlowe sur ses personnages ou au contraire de rejeter dans l'ombre les aspects de sa biographie qui cadrent mal avec les aspects de l'oeuvre qu'ils voudraient valoriser.'

Sans doute le poète tire-t-il de son expérience personnelle ce qu'il investit dans son oeuvre. Mais celle-ci cesse de lui appartenir aussitôt qu'elle devient texte dramatique et proposition de jeu. Elle acquiert de ce fait, par rapport à son créateur, une autonomie, une objectivité qui résultent de la pluralité des points de vue des personnages, et des jugements que le lecteur et le spectateur peuvent formuler sur eux. C'est en vain qu'on espère trouver un système d'explication continue permettant d'intégrer l'homme et l'oeuvre. Et même si la vie de Marlowe était beaucoup mieux documentée il subsisterait une rupture qui tient à la nature même de la création artistique, et plus particulièrement de la crêation théâtrale.

C'est la multiplicité des interprétations possibles qui assure la pérennité d'un texte. Mais il existe deux types de lecture, celle des spécialistes de la langue, de la civilisation, du théâtre élisabéthain, qui établissent le texte, l'élucident, le situent dans son contexte premier, celle des amateurs de 
poésie et de théâtre, ou des professionnels de la scène, qui souhaitent mettre en rapport les oeuvres du passé avec leur expérience personnelle et les préoccupations d'un public actuel. Bien entendu les lecteurs du premier type ne sont pas exempts de soucis de cet ordre et la nature même de leur besogne leur impose une perception plus aiguë du décalage historique. On ne saurait non plus s'attendre de leur part à une objectivité de tous les instants. Ils ont inévitablement leurs sympathies, leurs partis pris qui peuvent agir comme stimulants dans leur travail. Mais l'important pour eux est de savoir dans quelles limites ils peuvent exercer le jeu des interprétations. Car leur fonction est d'assurer une base solide à toute lecture, et de rendre clair le sens originel, celui qu'étaient susceptibles de recevoir les Elisabéthains, en fonction de leur culture populaire ou savante, de leurs habitudes théâtrales, de leurs croyances, des traditions et des changements dans leur société et leur conscience.

C'est par rapport à ces critères que j'ai essayé de formuler des questions susceptibles d'instaurer un débat.

a. Dans quelle mesure peut-on déduire les opinions de Marlowe de ses propos, de ses fréquentations?

b. Ses idées reflètent-elles un courant philosophique, et impliquent-elles une position à l'égard de la religion? Sa pensée forme-telle un ensemble cohérent dont on puisse retrouver les éléments dans ses oeuvres?

c. La structure dramatique de celles-ci permet-elle d'en donner une interprétation univoque, dans le sens de la subversion ou de l'orthodoxie?

d. Si l'on y discerne encore des schémas théologiques, en quoi se distinguent-elles de l'ancien théâtre religieux ?

e. Dans quelle mesure s'agit-il chez Marlowe d'un théâtre critique, exposant les conflits internes du monde chrétien, dénonçant la contradiction entre les aspirations et les réalités, les professions de foi et les actes?

Et j'ai examiné quelques uns de ces points dans mon introduction, dont le première partie était consacrée à la réputation de Marlowe, et la seconde à ses oeuvres dramatiques ${ }^{2}$. 


\section{La réputation et les idées}

Je choisirai comme entrée en matière un autre questionnaire, utilisé celui-ci par des notables et fonctionnaires chargés d'enquêter sur les opinions de Sir Walter Ralegh et de son entourage. Ce document est postérieur à la mort de Marlowe, mais il fait bien ressortir les dogmes essentiels dont la mise en question risquait d'attirer l'accusation d'athéisme $^{3}$. Qui, a votre connaissance, demandent les enquêteurs, a nié ou mis en doute l'existence de Dieu? Qui a contesté la Providence divine, la Création et la Fin du monde, le Paradis et l'Enfer, l'Immortalité de l'âme ? Qui a parlé contre la vérité de la parole divine dans l'Ancien et le Nouveau Testament ou qui a soutenu que sa Majesté ne devait pas croire aux Ecritures, ou les défendre en tant que doctrine de la foi et du salut mais par politique, et pour les fins du guuvernement civil ? ${ }^{4}$

Les apologistes du temps distinguent deux sortes d'incroyance que l'on retrouve ici. L'une résulte d'une curiosité intellectuelle trop ambitieuse qui conduit à l'examen téméraire des causes premières. L'autre consiste à considérer la religion comme une utile supercherie, destinée à maintenir le peuple dans l'obéissance et la soumission. Deux attitudes par conséquent, celle du philosophe ou du savant inspiré par l'orgueil, celle du politique qui se fonde sur la ruse. Leurs démarches sont distinctes mais elles peuvent se conjuguer pour ébranler le système des croyances. En effet comme il ressort du document cité, il suffirait de supprimer l'un des dogmes énumérés pour que soit compromis l'équilibre destiné à maintenir l'ordre social. Si la création dans le temps est contestée, si le monde n'a ni commencement ni fin, la crainte des châtiments futurs est vaine et ne peut plus servir à maintenir les hommes dans le droit chemin. Et il en va de même si l'on nie l'immortalité de l'âme.

Mais d'autre part réduire la religion au rôle d'instrument entre les mains du pouvoir civil, c'est faire reposer l'ordre social sur une illusion habilement entretenue et faire peser sur les gouvernants le soupçon d'indifférence aux principes moraux. On remarquera que les enquéteurs demandent si l'on a prétendu que la souveraine devait défendre la religion non pour que celle-ci accomplisse sa 
mission salvatrice mais pour qu'elle serve les intérêts du gouvernement civil. Cette formulation révèle un point vulnérable. Car Elisabeth, si elle est à la tête du gouvernement civil, est en même temps le chef de l'Eglise anglicane et la protectrice de la foi. Et du fait que l'autorité de la reine s'étend aux deux pouvoirs, l'Eglise se trouve dans une position de subordination. Or on connaît la situation de la chrétienté divisée, en ce siècle où les motivations politiques et religieuses sont inextricablement mêlées dans les conflits qui opposent nations et partis. Pour me limiter à des points qui touchent de près à notre sujet je rappellerai que l'expansion maritime et les visées coloniales entraînent les plus entreprenants des sujets d'Elisabeth à disputer à l'Espagne l'empire des océans, mais qu'en méme temps l'affrontement des deux monarchies apparait comme l'antagonisme de puissances qui défendent respectivement la cause du catholicisme romain et celle de la Réforme. Ce conflit se traduit par des opérations militaires, des manoeuvres diplomatiques, et par la guerre clandestine, qui est l'envers de la diplomatie, que se livrent les agents du gouvernement et ceux des catholiques anglais qui refusent de reconnaître un souverain réformé. Et pour qui a acquis la dure expérience de cet univers clos de l'espionnage et de la provocation, la contradiction entre la fin et les moyens ne peut manquer de devenir flagrante. Or c'est ce milieu que Marlowe a fréquenté, et c'est là qu'il a trouvé la mort.

Comme vous le savez les documents dont on dispose, bien que lacunaires, permettent d'établir que Marlowe avait pu, grâce à une bourse de l'archevêque de Cantorbéry, faire des études à Cambridge. Il s'était engagé à entrer dans les ordres après avoir passé ses examens mais le Conseil privé avait dû intervenir auprès des autorités de l'université pour justifier ses absences et dissiper la rumeur qu'il était l'un de ces étudiants qui avaient fui leur pays pour se rendre au séminaire des Jésuites de Reims, foyer des complots catholiques contre la couronne d'Angleterre. II est possible, mais non prouvé, qu'il ait joué un rôle de surveillance auprès des étudiants suspects, mais on peut tenir pour sûr qu'il était entré au service du gouvernement. On ignore si son activité d'agent secret s'est maintenue durant les brèves années de sa carrière de poète et de dramaturge. Par contre il ne fait aucun doute que Marlowe a passé le dernier jour de sa vie (le 30 mai 1593) en compagnie de trois agents connus dont l'un (Robert Poley) a joué un rôle de premier 
plan dans les services d'espionnage du gouvernement. On connait les faits. Sommé de comparaitre devant le Conseil privé, il est astreint par celui-ci à se présenter chaque jour jusqu'à nouvel avis. Mais dix jours plus tard il est blessé à mort et selon toute vraisemblance assassiné, bien que l'enquéte officielle ait conclu que celui qui l'avait frappé se trouvait en état de légitime défense. L'hypothèse d'une liquidation est soutenue d'une part par la réputation des trois personnages qui l'accompagnaient, de l'autre par la nature des informations reçues par le Conseil sur la conduite attribuée à Marlowe.

Les principaux documents relatifs à cette affaire ont été publiés pour la première fois par F.C. Danchin en 1913 et l'analyse la plus fouillée qui en ait été faite en France est celle de Pierre Lefranc dans son remarquable ouvrage sur Ralegh ${ }^{5}$. Ce dossier a été discuté bien des fois. Je me suis moi-même penché dessus à plusieurs reprises au fil des années, et toujours avec la même perplexité. Je voudrais éviter d'entrer dans trop de détails. Mais il est évident qu'on ne peut rien tirer de ce dossier qui soit utile pour l'histoire des idées sans tenir compte des distorsions qu'y subissent les opinions relatées dans le contexte d'une affaire d'espionnage fort enchevêtrée. Je rappellerai donc brièvement qu'il s'agit du rapport d'un indicateur (Richard Baines) sur l'athéisme professé par Marlowe, des pièces accusant un agent provocateur au service du gouvernement (Richard Cholmley) de reprendre à son compte les propos subversifs de Marlowe, et de jouer un double jeu auprès des catholiques qu'il est censé surveiller. Ces documents sont antérieurs à la mort de Marlowe, tandis que la lettre du dramaturge Thomas Kyd au Garde des sceaux (Sir John Puckering) leur est quelque peu postérieure. Kyd y repousse avec horreur l'idée qu'on puisse le soupçonner d'amitié avec un tel impie. Mais comme l'infortuné avait été arrêté et mis à la question peu auparavant pour une affaire distincte, il est probable qu'au cours de son interrogatoire il ait déjà porté les accusations que renfermait sa lettre.

Ces trois sources doivent être prises en compte pour préciser la nature et le degré de cohérence des opinions qui furent attribuées à Marlowe, et qui entraînèrent sa fin tragique. Par exemple il met en doute la chronologie biblique et le récit de la Genèse, faisant état d'antiquités 
antérieures à la création Adam. Quant à Moïse c'était un magicien. Initié aux arts des Egyptiens il lui avait été facile de tromper les Juifs, peuple grossier et ignorant, et il avait su, en prolongeant la durée de l'Exode, se débarrasser de ceux qui connaissaient ses secrets. Si Moïse est décrit comme un fondateur d'Etat habile à maintenir son peuple dans la superstition, le Christ est présenté comme un homme méprisable, de basse extraction, qui sans doute méritait le châtiment qui lui fut infligé. Ses Apôtres étaient des minables et le Nouveau Testament un écrit grossier. Déclarations propres à heurter la conscience chrétienne en son point le plus sensible, le croyance à l'Incarnation. Mais les arguments que rapporte Baines, peutétre en les simplifiant et les déformant, ne relèvent pas seulement du blasphème, et l'on y discerne les échos d'un débat d'idées. Nous allons y revenir mais il faut mentionner encore les déclarations attribuées à Cholmley, qui dans ses propos contre la religion se recommandait de l'autorité de Marlowe, affirmant que ce dernier avançait en faveur de l'athéisme des arguments plus forts que ceux des théologiens. Cholmley se plaisait à dire aussi que les membres du Conseil qu'il connaissait bien pour avoir marchandé avec eux le prix de ses services, étaient de profonds politiques qui, comme le prouvaient leur vie et leurs actes, ne pouvaient croire à l'immortalité de l'âme. La logique de ce discours paraît être que les ministres de sa Majesté, pour défendre la religion autorisée, recouraient à des pratiques contraires aux commandements de la foi qu'ils professaient. Leur conduite même devenait un argument en faveur de l'incroyance, et justifiait la duplicité à leur égard des agents qu'ils employaient.

A juger par ces dénonciations la propagande en faveur de l'athéisme était devenue un aspect majeur de l'activité de Marlowe, et la cause probable de son élimination. Mais pourtant le Conseil semble avoir été préoccupé, plus encore que par ces propos subversifs, par les complots qui se tramaient alors en Ecosse avec le soutien de Philippe II. C'est lorsqu'il était devenu évident que Cholmley, qui se vantait de jouer un double jeu, avait définitivement rejoint l'autre camp et prenait une part active à ces intrigues, que le Conseil avait donné l'ordre de le faire arrêter. Et il est très possible également que la décision de supprimer 
Marlowe ait été prise après que Kyd eut révélé que celui-ci se proposait de se rendre en Ecosse et cherchait à y entrainer des "personnes de qualité».

Les propos de Marlowe ne peuvent s'expliquer uniquement comme des provocations destinées à démasquer les adversaires du gouvernement. En dénonçant ses membres comme des politiciens sans foi il ne pouvait manquer de gagner la confiance des opposants catholiques, mais il ne pouvait que les horrifier par ses paroles impies. Ce qu'il disait de la supériorité de l'ancienne religion, à cause de la splendeur de ses cérémonies, suffisait d'autant moins à compenser l'effet de tels discours qu'il proposait aussi l'usage de tabac pour l'administration du sacrement.

Les paroles qu'on rapporte ne sont pas non plus des pièges destinés à démasquer des mécréants, ni des inventions des délateurs visant à l'accabler car nous avons aussi. sur ce point. le témoignage de poètes qui l'on connu, non seulement celui, contraint, de Thomas Kyd, mais celui de Robert Greene quil'exhortait au repentir. Mais d'autre part on s'étonne que Marlowe ait pu jouer le róle d'un espion auprès des catholiques et celui d'un agent double se préparant à changer de camp, et qu'il se soit livré parallèlement à une propagande contre les fondements de la religion. Non que cette attitude soit incohérente, elle parait relever au contraire d'une certaine logique ( si les défenseurs de la religion d'Etat utilisent de tels moyens on ne doit avoir aucun scrupule à les trahir. Et si ces moyens sont employés de part et d'autre dans la guerre secrète que se livrent les deux camps, les principes mêmes de la foi doivent être mis en question). Mais Marlowe n'a pu soutenir ouvertement, avec le véhémence qu'on lui prête, dans les milieux que l'on sait, une position aussi dangeureuse sans prendre les plus grands risques. Et c'est bien ce que la suite des événements a prouvé.

On peut discuter sur la nature de ses mobiles, mais les faits demeurent, même s'ils restent imparfaitement expliqués. Je continue à croire que ces opinions ont pesé lourdement sur la décision du Conseil à son égard, même si ses membres ont jugé l'affaire d'Ecosse la plus urgente. Et c'est pour se justifier de cette décision qu'ils ont transmis à la reine une copie du document le plus accablant pour Marlowe, le rapport Baines. Ils se sont également inquiétés 
de voir se répandre l'idée que la religion n'était qu'un instrument aux mains du gouvernement civil.

Et ceci nous ramène au questionnaire de l'enquête de Cerne que j'ai cité en premier lieu parce qu'il définissait clairement, selon des critères élisabéthains, l'athéisme philosophique et l'athéisme politique. L'enquête visait sans le nommer Ralegh et ses familiers. Elle était postérieure de dix mois aux poursuites contre Marlowe, mais elle se situait dans le prolongement de cette affaire. En effet Ralegh avait été accusé, dès 1592 , dans un pamphlet contre la persécution des Jésuites en Angleterre, d'entretenir une école d'athéisme dont le principal enseignant était un certain astronome et nécromancien, et où l'on tournait en dérision l'Ancien et le Nouveau Testament. Texte qu'on a souvent rapproché des propos attribués à Marlowe : Moïse n'était qu'un escamoteur et Harriot qui est au service de Ralegh est plus fort que lui; et à Cholmley : Marlowe a fait un exposé sur l'athéisme à Sir Walter Ralegh et autres. Ces citations sont les seuls indices d'un lien entre Marlowe, Ralegh et Harriot. Elles ne suffisent pas à prouver l'existance d'une «école» ou d'un cercle où la science aurait été associée à l'incroyance.

L'enquéte de Cerne n'apporta pas à cet égard de preuve décisive. Il s'agissait surtout de rumeurs, dont certaines visaient Harriot. Et l'un des témoignages les plus précis révélait que pour sa part Ralegh prenait plaisir à poser aux pasteurs de l'endroit des questions philosophiques embarrassantes. Il ne fut pas inquiété mais dans les années qui suivirent et jusqu'à son procés sa réputation d'athéisme fut utilisée par ses adversaires avec une vigueur croissante. Comme il fallait s'y attendre on ne trouve rien dans ses écrits qui rétrospectivement la justifie. Bien plus son Histoire du monde est pleine de professions de foi d'une d'orthodoxie inconststable. Et pourtant cet ouvrage majeur est de ceux qui appellent une lecture à deux degrés. On y retrouve un courant de pensée machiavelienne déià très perceptible dans ses écrits politiques. D'autre part, il y reprend à son compte l'idée de l'existence d'une prisca theologia, d'une révélation antérieure à l'Ancien Testament, et d'un savoir secret transmis par Hermès, auquel Moïse aurait été initié ${ }^{6}$.

Pour qui cherche à situer les propos de Marlowe dans 
le contexte philosophique et scientifique élisabéthain, il est significatif de le voir s'emparer lui aussi, pour l'interpréter à sa manière, de l'idée que Moise, prophète et législateur, avait été instruit dans la science des Egyptiens. Cette idée trouvait un appui dans les Actes des Apôtres (vii, 22) et dans les chapitres de l'Exode où Moïse fait usage contre les magiciens du Pharaon de la science acquise en Egypte. Les écrits hermétiques, traduits et commentés par Ficin, avaient contribué à valoriser la religion de l'ancienne Egypte et la croyance en l'efficacité des pratiques magiques. Pic de la Mirandole, dans son Oratio de homini dignitate et son lleptaplus, se réfère, en même temps qu'à cette tradition, à celle de la Cabale juive dont il fait remonter l'origine à Moïse. Il distingue dans les livres mosaïques le sens littéral destiné au peuple grossier, et le sens profond destiné aux initiés. De méme, pense-t-il, le Christ emploie des paraboles pour enseigner à la masse quelques principes moraux, et réserve la doctrine secrète à ses disciples. La majesté divine n'est pas mise en doute, au contraire elle est exaltée, mais seule une élite de l'esprit est admise à la contempler.

$\mathrm{Au}$ siècle suivant Giordano Bruno, qui compose en Angleterre plusieurs de ses dialogues, trouve son inspiration dans les traités de philosophie religieuse et de magie attribués à Hermès, et crus longtemps très anciens bien que se rattachant en fait à un courant néo-platonicien assez tardif. Mais le mystique des Eroici furori est aussi le polémiste plein de verve du Spaccio della bestia trionfante. Et c'est sur un ton mi-sérieux, mi-plaisant qu'il rapporte que lorsqu'il descendit du Sinai portant les tables de la Loi, Moïse, docteur ès sciences egyptiennes et magicien, revêtit pour convaincre le peuple qu'il était le représentant de l'autorité divine, une apparence surhumaine ${ }^{7}$. Moïse aurait donc agi en politique avisé. On ne sait si en écrivant ceci Bruno avait en mémoire des passages souvent cités du Prince (ch. VI) et des Discours sur Tite-Live (I, ch. IX) sur le rôle de la religion dans la création des Etats. Machiavel y déclare qu'il n'y a pas de légistateur qui n'ait eu recours à l'entremise d'un dieu pour faire accepter des lois nouvelles. II proteste que Moíse, seul, était vraiment l'exécuteur des ordres du Ciel. Cependant, ajoute-t-il, sa conduite n'était pas différente de celle des autres fondateurs d'Etats. La position de Machiavel est bien éloignée de celle de Bruno. 
Mais on voit comment a pu s'opérer un glissement de sens, que Marlowe exploite, de la philosophie mystique à la sagacité machiavélienne, sinon à la ruse machiavélique.

D'autre part lorsque Marlowe affirme que Harriot pouvait faire plus de choses que Moïse cela veut peut-être dire qu'un savant comme lui est plus fort qu'un quelconque magicien (a juggler). Cette phrase de Marlowe est à rapprocher de celle que rapporte $\mathrm{Kyd}$, selon laquelle les choses que l'on estime effectuées par la puissance divine pourraient aussi bien l'être par l'observation des hommes. Autrement dit ce qui paraît miraculeux pourrait être accompli gráce à l'observation des propriétés naturelles des choses. C'est dans des termes voisins que Ralegh définira plus tard une sorte de magie qu'il assimile à une philosophie de la nature et qu'il fonde sur la connaissance des effets et des causes. On sait combien il est difficile alors de faire le départ entre l'occultisme tel qu'on l'entend aujourd'hui, et combien sont encore floues les frontières entre le «naturel» et le «surnaturel». Les expériences auxquelles Ralegh se livre durant sa captivité s'inscrivent dans la tradition alchimique médiévale renouvelée par Paracelse. Mais sa pensée, on l'a vu, reste enveloppée de philosophie mystique.

Quant aux sciences mathématiques, dont on observe alors l'essor, elles sont loin d'être dégagées des spéculations platoniciennes et pythagoriciennes, qui d'ailleurs ont eu le mérite de promouvoir l'idée d'un ordre universel soumis aux lois de la proportion et du nombre. On sait que le plus éminent mathématicien de la génération précédant celle de Harriot, John Dee, utilisait aussi les nombres pour invoquer les esprits. L'exemple de ce disciple d'Euclide devenu adepte de la Cabale, et pratiquant une forme de magie particulièrement suspecte, est significatif, encore qu'il constitue un cas extrême. On conçoit que les savants les plus novateurs de ce temps aient été affectés par les courants de pensée, si vivaces au XVIe siècle, dont on ne sait s'il faut les appeler pseudo ou pré-scientifiques. Et que le travail de décantation ait été long et difficile. A juger du moins par la partie la plus aboutie de son oeuvre, Harriot se sentait peu attiré vers l'occulte, et représente les traits d'un scientifique d'un type nouveau.

Les études sur l'activité de Harriot et d'autres membres de l'entourage de Ralegh et du comte de Northumberland 
ont progressé, durant ces dernières décennies, grâce aux travaux des historiens des sciences et des idées ${ }^{8}$. Les causes de sa réputation d'impiété sont mieux connues, de même que la philosophie d'un esprit curieux comme Nicholas Hill, et celle du mathématicien Walter Warner, qui fut l'associé de Harriot et son continuateur. ${ }^{9}$

L'essor scientifique élisabéthain. faut-il le rappeler, est stimulé par l'expansion maritime et la colonisation. Pour employer le langage actuel la recherche fondamentale et la recherche appliquée sont solidaires. Aux besoins de la navigation répondent les progrès des mathématiques, de l'astronomie, de l'optique, mais aussi le perfectionnement des instruments, des cartes, la multiplication des observations systématiques et quantifiées.

$\mathrm{La}$ théorie de Copernic est couramment admise dans ces milieux et elle est exposée de bonne heure dans les ouvrages de Robert Recorde, et surtout de Thomas Digges. La «révolution copernicienne» devait à plus ou moins long terme remettre en question la représentation cosmique traditionnelle qui est une combinaison de la cosmologie ptoléméenne et de la physique aristotélicienne. La théorie du mouvement des planètes autour du soleil était présentée par Copernic non seulement comme une manière plus simple et plus élégante de rendre compte des phénomènes mais comme une description de la réalité physique. Son succès dépendait donc des observations qui viendraient la confirmer. Et à cet égard on doit distinguer deux phases dont la première est celle des spéculations et la seconde celle des observations décisives, effectuées à l'aide d'instruments d'optique par Galilée en Italie mais aussi par Harriot en Angleterre. Ceci nous conduit aux alentours de 1610. Et il faut se rappeler que Marlowe n'a rien connu de ce développement.

En substituant le Soleil à la Terre comme centre du Monde Copernic battait en brèche la notion aristotélicienne d'une différence qualitative entre la région céleste et la région sublunaire. Selon Aristote les corps de la première étaient inaltérables alors que ceux de la seconde étaient soumis à la génération et à la corruption. Et de plus les uns et les autres étaient soumis à des mouvements différents. Le système nouveau permettait donc du supposer l'uniformité de la matière et des lois du mouvement. C'est ce 
qu'avait bien compris Bruno dont on sait qu'il a composé en Angleterre plusieurs de ses écrits, dont le dialogue $L a$ Cena delle Ceneri où il défend Copernic contre les champions de la vieille scolastique.

Son apport est celui d'un philosophe exalté plutót que celui d'un scientifique. Comme il ressort d'autres ouvrages de sa main le système solaire n'est qu'un point dans l'univers qu'il conçoit, qui est infiniment étendu dans le temps et dans l'espace. La matière qui le constitue est faite d'atomes inaltérables, mais susceptibles d'être organisés en d'infinies combinaisons. Cette représentation de l'univers n'est pas purement matérialiste. Elle se fonde au contraire sur une méditation concernant les attributs de la divinité dont la puissance infinie ne peut se manifester que par une création incessante, et illimitée. Les sources de cette vision se trouvent à la fois dans le néo-platonisme, dans la philosophie de Démocrite, d'Epicure et de Lucrèce, et dans des spéculations médiévales sur l'infini et l'atome.

Les voyages d'exploration sont un autre stimulant de la pensée de Bruno. Sa philosophie est évidemment en contradiction avec l'idée d'une création dans le temps et ex nihilo. Mais voici que le récit de la Genèse est mis en question par la découverte dans le Nouveau Monde non seulement d'autres races humaines, mais de vestiges de civilisations très anciennes. Dans son dialogue lucianesque, le Spaccio, il conteste la diffusion de la race humaine dans les divers continents à partir de la seule lignée d'Adam. Ceci recoupe évidemment les propos attribués à Marlowe sur les contradictions de la chronologie biblique faisant remonter la création du monde à quelques milliers d'années alors qu'il existe des antiquités indiennes beaucoup plus anciennes. Et ceci recoupe aussi les attaques de Thomas Nashe contre les mathématiciens qui prétendent prouver l'existence d'une humanité préadamique. Harriot, qui dans son rapport sur la Virginie fait preuve d'une assez grande largeur de vue dans ses jugements sur les sociétés indiennes qu'il a observées, était bien l'homme à s'intéresser à ce problème mais il n'y a pas traces dans ses papiers de calculs sur la chronologie du Nouveau Monde.

L'influence de Bruno en Angleterre est sensible dans un livre (Philosophia epicurea, democritiana, etc.) de Nicholas Hill qui était au service de Northumberland et que Harriot devait bien 
connaitre. Hill y reprend à son compte la philosophie de la nature de Bruno. Il fonde la religion du savant sur l'intelligence des lois qui régissent l'univers. Et l'observation de l'uniformité et de la simplicité des opérations de la nature engendre dans son esprit la constance. Mais ni cette religion, ni l'éthique qui en dérive ne sont à la portée du vulgaire. Celui-ci, pour bien se conduire, a besoin de croire aux châtiments dans une autre vie, alors que celle-ci est inconcevable dans un univers infini.

La réputation d'impiété de Thomas Harriot a été longtemps entretenue par des ecclésiatiques, parmi lesquels l'un de ses disciples Nathaniel Torporley. Ils lui reprochaient d'avoir fait sien le principe démocritéen Ex nihilo nihil fit (rien ne se fait de rien) et admis l'existence d'une matière inaltérable composée d'atomes, ce qui impliquait la négation du récit de la Genèse. Les deux questions étaient liées, c'est pourquoi Torporley avait entrepris une réfutation de l'atomisme de Harriot, où il défendait le principe adverse Ex nihilo omnia.

Selon une tradition recueillie par Aubrey le cancer dont Harriot était mort fut considéré par les théologiens de son temps comme un jugement de Dieu. Cependant le reproche que lui font ceux qui l'ont connu n'est pas d'avoir été un athée, mais d'avoir soutenu une théologie philosophique téméraire et contraire aux Ecritures. En dehors de l'hypothèse atomiste on trouve dans ses papiers peu de traces précises d'une telle philosophie. Sur ses convictions religieuses on peut citer notamment une lettre très digne adressée à son médecin où il exprime sa foi en la toute puissance de Dieu, mais aussi en la médecine instituée par Lui, et en le médecin qui dans l'exercice de sa science agit comme le ministre de Dieu. Harriot déclare aussi se soumettre avec constance à tout ce qui lui arrivera, conformément à la Providence, mais sans renoncer pour autant à agir contre la maladie. Profession de foi importante car elle affirme la conformité de la science avec l'ordre divin, mais aussi la dignité de la science fondée sur la connaissance des effets de la nature. Elle n'est pas en contradiction avec les principes de la religion officielle, mais elle n'est pas en désaccord non plus avec les principes d'une philosophie comme celle de Bruno.

La différence de Harriot avec Bruno résiderait plutôt 
dans une conception plus rigoureuse de la science. Bruno est un visionnaire qui découvre dans le système de Copernic une confirmation des spéculations sur l'infinité de l'univers. Harriot est ouvert aux hypothèses mais il est avant tout l'homme du calcul et de l'observation conduite avec patience et rigueur, comme en témoignent par exemple ses tables de réfraction. S'il propose à Kepler une explication atomiste de ce phénomène, c'est à titre d'hypothèse. Ses observations des corps célestes à l'aide d'un télescope (aspérités de la surface lunaire, taches du Soleil, satellites de Jupiter) sont contemporaines de celles de Galilée et, comme elles, contribuent à renforcer la théorie de Copernic et à ruiner la physique cosmique d'Aristote. Mais l'héliocentrisme ne suffisait pas, à lui seul, à éliminer les vestiges d'une cosmologie archaique. Kepler, qui avait découvert les lois du mouvement des planètes, croyait encore à l'existence de la sphère des étoiles fixes qui limitait de toute part le cosmos et sur la voûte de laquelle les étoiles étaient plantées, toutes à égale distance du centre. Pour reprendre l'expression d'Alexandre Koyré l'éclatement de cette sphère des fixes constitue une étape décisive dans le développement d'une nouvelle astronomie. Bruno niait l'existence de cette sphère et voyait dans les étoiles autant de soleils dont le nombre était illimité et dont les plus lointains, à cause de leur distance, échappaient à la vue. Harriot, et d'autres observateurs de son entourage, prennent parti contre Kepler. Mais l'infinité de l'univers n'est pas démontrable et ce qui les intéresse surtout c'est l'absence de limites dont ils espèrent avoir confirmation grâce à la découverte de nouveaux corps célestes que le progrès des instruments d'optique permettrait de rendre visibles.

En bref je crois que Harriot est plus proche de Galilée que d'un prophète comme Bruno, ou même un savant comme Kepler qui se représente le cosmos comme une image et un symbole de la Trinité. Ce qu'il faut retenir c'est que ce développement est très postérieur à la mort de Marlowe. Certes de son vivant Harriot pratiquait déjà les mathématiques, l'astronomie et l'optique, et se livrait sans doute aussi à des spéculations sur la philosophie de la nature. Mais je me demande dans quelle mesure Marlowe a compris son activité scientifique.

Ce qu'il parait avoir voulu faire c'est jeter un discrédit 
sur les livres sacrés. C'est ainsi qu'il a mis en question la chronologie biblique, et fait de Moise un fondateur d'Etat selon Machiavel, qui subordonnait la religion à la politique. Ces propos étaient applicables à la situation élisabéthaine, et prennent tout leur sens si on les rapproche de ceux qui visent la conduite du gouvernement et révèlent la mentalité de ses agents secrets dans les conflits de religion. Et c'est vraisemblablement pour cela que le Conseil de la Reine a tenu Marlowe pour dangereux.

Le dossier concerne, rappelons-le, le dernier Marlowe, postérieur aux pièces, et ne correspond pas à un état d'esprit qu'il aurait par la suite dépassé. Il ne s'agit pas d'accusations vagues, et l'on ne peut aisément faire abstraction de ces documents. Mais il n'est pas aisé non plus de les faire entrer en ligne de compte dans l'interprétation de ses oeuvres. Dans les pages qui suivent je me suis limité, pour des raisons déjà dites (voir plus haut n. 2), à une confrontation des deux grandes figures de Tamerlan et de Faust, un examen de la première et de la dernière oeuvre destinée au théâtre public ${ }^{10}$. Pièces majeures pour l'exposé des grands themes des aspirations et de la connaissance, mais qui ne rejettent nullement dans l'ombre Le Juif de Malte ou Edouard II qui ont leur problématique propre mais où l'on retrouve des constantes de la dramaturgie marlovienne.

\section{Aspirations et connaissance dans Tamburlaine et Faustus}

\section{For Tamburlaine, the scourge of God, must die.}

Dans Tamburlaine, Marlowe tient une gageure qui parait impossible : soutenir l'intérêt, voire l'admiration, pour un héros grandiloquant: "threatening the worid with high astounding terms». Sa rhétorique n'est pas creuse : tout ce qu'il conçoit se réalise, son verbe se traduit en actes, irrésistiblement, comme s'il était un dieu. Les images proliférantes de ce discours se réfèrent à un système du monde qui mêle cosmographie et mythologie mais qui reste cohérent dans sa fonction dramatique. On peut suivre l'itinéraire de ses conquêtes terrestres dans l'Atlas d'Ortelius (et comparer ses appetîts à ceux des puissances colonisatrices qui se disputent alors les continents). Le firmament, demeure du dieu souverain, est le lieu de ses 
aspirations et l'objet de ses révoltes. La région inférieure du ciel, domaine des météores, est violemment pertubée par ses guerres. Enfin, l'enfer paien d'où surgissent les forces de destruction et de mort est associé à ses menaces comme aux malédictions de ses adversaires. Ainsi Tamerlan emplit et ébranle l'univers entier.

Au départ il n'a d'autre justification que son audace, sa force de persuasion qui le fait reconnaître comme favorisé, maitre de la fortune, désigné par le Ciel. Le premier monologue où il définit sa relation avec la divinité est longuement préparé. Il survient au moment où ce chef de bande, après avoir aidé Cosroe à usurper le trône de son frère, s'est retourné contre lui, et l'a vaincu. Tamerlan célèbre l'aspiration de l'âme humaine, incitée par la contemplation de l'architecture du monde à poursuivre inlassablement un avoir infini. Démarche de l'esprit qui ne serait pas opposée aux enseignements de la théologie si l'objet de cette exaltation n'était une couronne «terrestre» et non, comme on l'attendait, "céleste» " . On discerne dans ce passage un écho des livres d'Hermès et des commentaires qu'ils ont suscités : noblesse de l'homme dont le génie comprend l'univers, et fait de lui l'égal des dieux. L'opposition céleste - terrestre, contemplation - action, n'est d'ailleurs pas irréductible dans cette philosophie mystique qui distingue une voie ascendante pour accéder au divin et une voie descendante pour attirer sur terre les bienfaits du ciel. Mais pour le héros la connaissance n'est rien sans la possession, et la violence est l'unique moyen du pouvoir. Mû par la soif de régner il pourrait devenir roi pour maintenir sur terre un ordre semblable à celui qu'un dieu maintient dans l'univers. Mais son modèle est Jupiter, qui a usurpé le trône de son père, et qui a dû faire la guerre aux Titans rebelles à son autorité. A ses yeux le principe qui gouverne la nature et qui propulse ses désirs est celui de la guerre. C'est pourquoi le vers The sweet fruition of an earthly crown. loin de correspondre à une chute dans l'inspiration, souligne avec ironie une contradiction sur laquelle est construite tout le drame. Ce discours acquiert tout son sens du fait qu'il est proféré tandis que Cosroe ensanglanté agonise. Et par la suite les plus hautes envolées du héros seront associées aux spasmes de la cruauté.

Seule 7énocrate serait susceptible de fléchir sa volonté 
implacable. Ils forment un couple divin dont les figures sont opposées et complémentaires. La beauté surhumaine et le pouvoir de séduction de Tamerlan, du moins dans sa jeunesse, avant que les travaux guerriers n'aient durci ses traits, et que la fureur n'ait altéré sa complexion, sont capables d'émouvoir ses ennemis mêmes ${ }^{12}$. Zénocrate se voit, et il la voit, en reine du ciel, épouse et soeur de Jupiter $^{13}$. Mais la beauté de celle-ci, et l'amour qu'elle lui inspire, désignent un autre chemin vers le divin que la conquête, une autre sorte d'aspiration dont il fait l'expérience, mais devant laquelle il se sent vulnérable, et à laquelle, finalement, il résiste. Ceci nous vaut les sommets poétiques de l'acte $\mathrm{V}(1, \mathrm{~V}$, ii, 72-115). Mais le contraste entre l'action et cette méditation merveilleuse est devenu. non sans raison, un lieu commun de la critique. Les cadavres des Vierges de Damas ont été hissés sur les remparts et il vient d'ordonner que le reste de la population soit passé par les armes. Ft il interrompt le cours de ses pensées pour se préoccuper de nouveaux moyens d'humilier Bajazet. Mais 7énocrate elle-méme est captive, elle s'afflige du destin de son peuple et tremble pour son père. Sa beauté troublerait moins le héros s'il ne la voyait, les cheveux épars, essuyant ses larmes. La souffrance de l'aimée s'ajoute au sang des victimes pour aviver cette flamme érotique qui ne va pas sans rappeler une cruauté racinienne ${ }^{14}$ sinon sadienne.

Tamerlan se justifie du massacre des innocents par la nécessité d'un principe cosmique de destruction qu'il incarne, et sur lequel il fonde les lois de la guerre. Il invoque ses règles martiales, as peremptory As wrathful planets, death or destiny, sa "discipline» et même sa "chevalerie». Il ne s'agit pas des caprices sanglants de quelque: despote oriental. Sa rigueur, illustrée par le symbole des tentes blanches, rouges, et noires, est extrẻme mais ne diffère pas, dans sa méthode, de la pratique de l'Occident, au siècle de Marlowe, et particulièrement dans la guerre de siège. Les menaces, sévérement graduées en vue de leur efficacité tactique, ont en même temps un aspect cérémoniel où le souverain en armes apparait comme le dispensateur d'une roide justice. Les Vierges de Damas l'ont bien compris, elles lui apportent l'of frande du laurier, et font l'éloge de ses vertus, consacrées par le ciel. Mais leur appel à la clémence survient trop tard et il leur reste le rôle de victimes 
sacrificielles.

Quant au traitement de Bajazet et de Zabina, il n'est même plus justifié par la nécessité d'un exemple. Pour mieux jouir de son triomphe, Tamerlan inflige aux vaincus les tourments moraux qui les réduisent au désespoir. Zénocrate elle-même a cédé à la tentation d'humilier la reine déchue. Elle s'en repentira devant les cadavres des époux qui se sont donnés la mort. Et elle implorera le pardon du Ciel pour Tamerlan et pour elle. Cette capacité d'éprouver de la compassion, et de redouter la démesure, sont caractéristiques du personnage, et l'opposent au héros. On retrouvera cette disposition d'esprit dans la scène de sa mort. Mais malgré le culte que Tamerlan lui voue, son rôle se réduit du commencement à la fin à la patience et la soumission.

Observe-t-on, en passant à la deuxième partie, un changement dans la mentalité du protagoniste ? y a-t-il eu perte d'une vision ? Il faut rappeler d'abord que les traits de cruauté et de fureur qui iront en s'exaspérant encore étaient déjà très accusés dans la première partie. D'autre part, la scène de la mort de Zénocrate (2 II iv) est empreinte d'un lyrisme aussi exalté que celle où le héros célébrait sa beauté. Le vers y atteint même une musicalité jamais égalée chez Marlowe. La musique que la reine demande, après avoir reconnu la fragilité de sa condition mortelle et exprimé l'espoir d'une autre vie, est à l'image d'une âme accordée à l'harmonie des sphères célestes et des choeurs angéliques. Pour le héros l'expérience de la destruction de la beauté est plus poignante encore que celle de sa révélation. Et cette fois encore il réagira à la blessure de l'amour par des actes de cruauté. Non seulement il retiendra auprès de lui la reine morte au lieu de la rendre à la terre, mais il instaurera un rite funèbre et lui offrira des villes incendiées en sacrifice.

La composition d'une suite dépendait évidemment du succès d'une pièce conçue à l'origine pour se suffire à elle-même. Mais les deux parties s'enchaînent remarquadblement bien comme si l'auteur en avait eu d'emblée une vue d'ensemble, et l'on remarque de nombreuses correspondances qui lient la seconde à la première. Tamberlaine n'est pas une simple transposition dramatique des chroniques. I a page de titre présente les deux parties 
comme des «discours tragiques» et le prologue de la première la définit comme un "miroir tragique», c'est-àdire comme un exemple des vicissitudes de la fortune et de la condition instable des grands. Inconstance qu'illustre dans cette partie le destin des victimes du conquérant alors que celui-ci marche de victoire en victoire. Il en va tout autrement dans la seconde partie, telle que la décrit son prologue : Where death cuts off the progress of his pomp. And murderous Fate throws all his triumphs down. $\mathrm{Ce}$ concept de triomphe livre une des clés de l'oeuvre, et contribue à lui donner sa forme. C'est lorsque Cosroe s'apprète à faire une entrée pompeuse dans sa capitale qu'il décide de s'emparer du royaume de son allié, And ride in triumph through Persepolis. (1, II, v, 48-54). L'élément spectaculaire de l'oeuvre, et son caractère processionnel, sont bien mis en évidence dans les indications scéniques. Les deux modèles en sont le triomphe militaire romain avec ses enseignes, son butin, ses captifs humiliés, et le triomphe allégorique selon Pétrarque : ici celui de la mort. Les batailles et la célébration des succès scandent la progression irrésistible d'une armée dont les étapes sont marquées par une série de tableaux mémorables par leur excès même : Bajazet dans la cage de fer, les costumes et les oriflammes écarlates pour le banquet, noirs pour le massacre des Vierges, le catafalque de Zénocrate, le char traîné par les rois.

Jusqu'à l'ultime scène Tamerlan conserve la victoire. Il ne connait d'autre défaite que la mort. Celle de Zénocrate avait été pour lui une épreuve et non un avertissement. Par défi il avait refusé de se séparer d'elle et associé son corps embaumé à ses triomphes. Mais sa propre agonie lui fait reconnaitre la nécessité, et sa condition d'homme. Il lui reste l'espoir de survivre dans sa descendance, et il confie aux deux fils qui lui restent (ayant tué le troisième) la tâche de terminer ses conquêtes et de gouverner ses Etats. Cette tâche leur paraît trop lourde. Mais rien dans le dénouement n'indique qu'ils seront incapables de s'en acquitter. Et l'on ne peut faire entrer en compte le fait historique que son empire fut sans lendemain. Même si l'on ne veut voir dans le protagoniste qu'un Fléau de Dieu utilisé par la Providence et détruit par elle, on est contraint d'admettre que s'il est éprouvé il ne subit pas la défaite 
écrasante d'un Richard III ou d'un Macbeth.

Les rapports du héros avec la divinité sont complexes. Ils sont définis par des déclarations, les siennes, celles de ses alliés, de ses ennemis, qui doivent être jugées en fonction des événements de la pièce, et de l'idéologie religieuse élisabéthaine. On discerne un premier niveau d'explication qui attribue ses victoires à une conjonction favorable des étoiles, agissant à la fois sur le cours des événements et sur le tempérament du conquérant, si bien que l'ardeur et l'irascibilité qui l'ont soutenu dans ses actions guerrières finissent par le consumer, et priver son âme des esprits qui lui sont nécessaires (comme l'explique son médecin : $2, \mathrm{~V}$, iii, 81 et s.). Mais l'influence des astres, et celles des humeurs, sont rarement évoquées seules. Et Tamerlan, dans ses discours, ne conçoit pas l'àme, et son destin, indépendamment de la divinité. C'est au moment où il immole un de ses fils qu'il profère les affirmations les plus audacieuses. La raison de ce meurtre est que lui, Tamerlan, a reçu de Jupiter une âme de nature divine tandis que Calyphas, bien qu'il soit de la même chair que le héros, a reçu une âme débile (2, IV, i, 111 et s.). Geste et paroles égalements sacrilèges car il justifie sa révolte par son identité avec le Créateur : ce sont les vertus qu'il a reçues qui le font s'insurger contre lui. On a vu qu'au début de sa carrière de fondateur d'empire il invoquait le précédent de Jupiter usurpant le trône de son père mais aussi châtiant les Titans rebelles. Dans son langage le nom de Jupiter désigne tantôt le dieu de la mythologie gréco-latine, et tantôt le Dieu supreme, The chiefest God, first mover of that sphere. (1. IV, ii, 8) et une ambiguiité subsiste entre les deux significations. D'autre part il se déclare Fléau de Dieu : I that am term'd the Scourge and wrath of God (1, III, iii, 44) selon un concept d'origine biblique et patristique mais employé ici dans le contexte de la guerre contre l'Islam, si bien que Tamerlan, livrant bataille à Bajazet, apparait comme le champion des chrétiens réduits à l'esclavage. Et la représentation de sa victoire sur les Turcs, et des humiliations infligées à leur empereur, tire une partie de ses effets de l'hostilité éprouvée par le public du temps à l'égard des «infidèles». Mais dans la seconde partie-la perspective change. Sans doute Tamerlan y demeure le fléau des Musulmans et met en fuite le fils de Bajazet 
après avoir vaincu et humilié ses alliés. Cependant dans une action secondaire, mais où figure l'un d'eux, se trouve posée une question grave : doit-on tenir parole aux «infidèles»? Marlowe transpose ici des événements qui n'appartiennent pas à l'histoire de Tamerlan mais à celle des princes chrétiens guerroyant contre les Turcs. Sigismond de Hongrie, en violant le traité qu'il a signé avec Orcanes, enfreint une loi universelle de l'éthique religieuse. Le roi d'Anatolie, attaqué par surprise, s'indigne de la perfidie des chrétiens et demande au Christ, s'il est le fils du Dieu vivant (everliving Jove), de lui accorder la victoire.

La conscience protestante était sensibilisée à ce thème de l'indignité des Chrétiens et Luther lui-même avait pris position pour le respect de la foi jurée aux infidèles. Il est vrai que les Réformés avaient intérêt, jusqu'à un certain point, à s'opposer à l'esprit de croisade dont le SaintSiège tirait avantage et à affaiblir les puissances catholiques menacées par les Turcs. Mais ce que ces scènes mettent en relief, c'est la duplicité des conseillers de Sigismond qui recommandent une "politique» contraire aux "professions de foi" des Chrétiens et tiennent pour "superstitieuse" l'extension aux infidèles des préceptes de la vraie foi. "Policy", "profession", "superstition" sont des termesclefs qu'on retrouvera dans Le Juif de Malte, où la confrontation ne sera plus entre deux, mais trois religions. Déjà dans ces scènes de Tamburlaine on discerne un appel à l'esprit critique, et la relativité des croyances est suggérée. Ainsi dans la scène du serment Sigismond et Orcanes invoquent respectivement le Christ, «fils de Dieu» et Mahomet "ami de Dieu»: Et le prophète se voit attribuer, lorsqu'il quitte le monde, une ascension aussi miraculeuse que celle du Messie (2, I, ii, 50-65). Cependant, après le viol de ce serment, Orcanes attribue sa victoire au Christ qui l'a exaucé. Mais l'un de ses alliés se déclare sceptique : "Tis hut the fortune of the wars, my lord. Whose power is often prov'd miracle (2, ii, iii, 31-32)

L'invocation d'Orcanes à un Dieu de justice est généralement considérée comme le passage exprimant la conception la plus élevée de la divinité dans Tamburlaine, sinon dans toute l'oeuvre : (...) he that sits on high and never sleeps, Nor in one place is circumscriptible, But evervwhere fills every continent With strange infusion of his sacred 
vigour (2, II, ii, 49-52). Et s'il y a un rapprochement à faire avec Bruno c'est bien ici. Mais l'idée d'une religion universelle en laquelle se résolvent les croyances particulières est loin de dominer. Orcanes lui-même sera réduit à trainer le char de celui qui se déclare Fléau de Dieu, et si cette mission exterminatrice est providentiellement permise, voire téléguidée, on ignore à quelle fin intelligible, et selon quelle justice. Sa propre justification, Tamerlan la donne aux rois turcs qu'il a vaincus. Elle se fonde sur une conception fort sombre du pouvoir séculier qui associe des vues pessimistes sur la nature pécheresse de l'homme et sur la nécessité du maintien d'un ordre sévère, et considère le glaive du souverain comme l'instrument de la colère divine. Au XVle siècle cette conception trouve une expression rigoureuse chez Luther et dans les homélies de l'Eglise anglicane ${ }^{15}$.

Tamerlan est-il finalement châtié et détruit après avoir exécuté les desseins de la Providence ? autrement dit existet-il un enchainement de cause et d'effets qui conduise à un tel dénouement? Certes ses ennemis vaincus prédisent que sa violence est devenue si excessive qu'ils seront bientot vengés, et qu'il sera consumé par une fièvre qu'ils décrivent dans les mémes termes que le médecin qui en fera le diagnostic ${ }^{16}$. Mais il ressent les premières atteintes du mal peu après avoir fait brûler le Coran et défié Mahomet de venger les musulmans qu'il a fait massacrer. C'est alors que s'adressant à ses soldats il les exhorte à chercher une autre divinité à adorer : The God that sits in heaven, if any god, For he is God alone, and none but he (2, V, i, 199-201). $\mathrm{Ce}$ bref passage est un exemple des contradictions auxquelles achoppe toute tentative d'explication univoque de l'enchaînement dramatique. Le Dieu suprême, dont Orcanes avait eu la vision, est identifié avec celui dont Tamerlan est le fléau, et à ce titre le héros est l'ennemi implacable des mahométans. Mais sa religion, qui selon les chroniques était islamique, n'est pas spécifiée dans l'oeuvre, oú le dieu qu'il sert pourrait être celui des chrétiens, puisque c'est leurs ennemis qu'il persécute. Cependant sa déclaration s'accompagne d'une sérieuse clause de réserve : "le Dieu qui trône dans le ciel, s'il y a un dieu", dont la tournure rappelle certains propos rapportés sur Marlowe: That if there be any god or any good religion. then it is in the papists ... etc. 
D'autre part peu avant de défier Mahomet, dans la scène où il immole son fils, il a bravé et menacé le Dieu suprême. L'incohérence de ses déclarations a été interprétée comme le signe d'un délire qui va croissant et finit par ravager son corps et son esprit. Et sans doute cette fin peut-elle être considérée comme une rétribution. Mais l'idée qu'il est le glaive du Seigneur fait partie de ce délire, et rien ne dit qu'elle correspond à une réalité. Lui seul s'attribue ce rôle de fléau, et sur la scène nulle voix doctrinale ne vient le spécifier. Si la Providence intervient elle reste silencieuse. Cependant on assiste bien à une sorte d'expiation. Et rien n'est plus impressionnant que cette destruction du personnage par lui-même, cette logique intérieure de la maladie, cette critique interne de la démesure, du déséquilibre et de la difformité qui résultent d'une perversion des aspirations.

Cependant Marlowe avait misé dès le début sur la fascination exercée par son personnage, et écrit une suite pour répondre à la demande d'un public avide d'actions et de paroles étonnantes. Il ne pouvait donc le laisser se désintégrer sans le rappel final d'une véritable grandeur. Dans la dernière scène ses fils et ses généraux fidèles forment un choeur tragique associant l'éloge à la déploration. Il parait sortir du délire qui le fait s'insurger contre Dieu et la Mort qui le tourmentent. Il finit par reconnaitre, dans "le monstre sans yeux" le monarque de la terre, et il accepte la nécessité. Il songe à la transmission et à l'extension de ses conque tes et il conseille à ses fils, pour gouverner le char de l'empire, de suivre l'exemple d'Apollon, et non de Phaéton.

Cette dernière phase ne peut cependant effacer l'impression de l'aggravation à travers l'ocuvre d'une fureur dévastatrice qui ronge celui qu'elle possède en méme temps qu'eile ravage les royaumes. Les contradictions quant à la nature du personnage et la logique des événements me semblent résulter, plutôt que d'un vice de construction, d'un conflit entre des concepts, des idéologies, dont l'oeuvre est la transposition dramatique. Hlles témoignent de la préoccupation des Elisabéthains à l'égard du chef armé dans ses fonctions de conquéte et de répression. Tamburlaine est le miroir d'un monde où sévit la violence, et l'anatomie d'un homme de guerre qui n'est pas sans rappeler le prince 
selon Luther et selon Machiavel. Cette oeuvre dont le héros se prend pour un dieu méchant met en évidence la forme "paranoïa» dont s'accompagne le pouvoir tyrannique. L'analyse qu'elle nous donne de sa maladie, en termes élisabéthains, est à ce point de vue fort efficace. Mais elle va beaucoup plus loin qu'une étude de cas. Elle montre à la fois Tamerlan et son délire, qui s'organise par distorsion et mise en conflit des doctrines philosophiques et religieuses prévalentes. Le mal est la composante majeure de cet univers subverti. La cruauté est associée à l'exaltation amoureuse, à la perception de la beauté, aux plus hautes aspirations. La violence est un principe de la nature, dans un cosmos où la lutte l'emporte sur l'harmonie. Elle est aussi un attribut de la divinité, le seul d'ailleurs qui apparaisse, car la Providence ne se manifeste que par les épreuves et les châtiments qu'elle impose. L'invocation à une instance suprême, à un Dieu de justice, vigilant et bienveillant, n'est pas le fait de Tamerlan mais d'un prince musulman qui sera finalement subjugué.

\section{Yet art thou still but Faustus, and a man.}

Alors que Tamerlan ne reconnait la nécessité de la mort qu'au terme de sa carrière, la certitude de la mort est pour Faust un point de départ. On sait que la pièce de Marlowe se modèle sur la version anglaise d'une chronique allemande, The Historie of the damnable life, and deserved death of Doctor Iohn Faustus, dont le titre indique assez qu'elle se veut édifiante et que la punition de son héros est exemplaire. Il s'agit d'un personnage réel, figure de clerc itinérant, dont la légende s'est enrichie d'éléments appartenant au folk-lore et à la culture populaire. Après s'être égayé de ses aventures, le lecteur est invité à tirer la leçon de ses transgressions. Ce qui touche à la morale et la damnation sera retenu dans la pièce, mais seront aussi maintenues les scènes comiques ayant pour thèmes les facéties du magicien, les diableries et la satire de la «superstition» à la cour pontificale. Dès lors que, s'inspirant de la chronique, il entreprend d'écrire The Tragical History of the life and death of Dr. Faustus, Marlowe se voit contraint d'adopter une forme dramatique composée d'episodes répartis sur toute une vie, bien que 
les moments proprement tragiques soient ceux du pacte, des crises de repentir non suivies d'effets, et enfin de la damnation. Il est amené à distinguer deux niveaux dans le déroulement du temps. celui d'une existence qui s'accommode d'un compromis avec le monde et le diable, et s'installe dans l'oubli des fins dernières. Celui d'une conscience qui, périodiquement, se réveille et tente de se libérer. Le premier est nécessaire au second car il suggère une épaisseur dans la durée, et qu'il meuble celle-ci d'activités dont le caractère dérisoire contraste avec la gravité de l'enjeu. Quant à la fonction du comique elle est, dans la chronique comme dans tout ouvrage didactique, d'ajouter un peu de miel pour faire passer l'amertume du remède, mais aussi de provoquer un défoulement en permettant au lecteur de participer sur le mode du rire à la transgression du héros, quitte à lui faire éprouver ensuite l'horreur du châtiment.

Les témoignages anciens, selon lesquels de vrais démons se seraient mêlés aux acteurs lors de certaines représentations du Dr. Faustus, montrent assez l'ambivalence des émotions suscitées alors par de tels spectacles. Et bien entendu derrière la pièce de Marlowe il y a toute la tradition du théâtre médiéval, avec son aspect doctrinal et la théâtralisation des dogmes (la Chute et la Rédemption, le Salut et la Damnation), avec son emploi des structures de la psychomachie (le Bon et le Mauvais Ange et la représentation symétrique du Paradis et de l'Enfer), avec enfin ses aspects grotesques formant la contrapartie du drame.

Faust est sans doute, des tragédies élisabéthaines, la plus fortement marquée par cet héritage médiéval. Mais destinée aux scènes publiques où l'oeuvre théatrale est devenu une commodité répondant à des besoins que le prix d'une place peut satisfaire, elle n'est pas directement mêlée à une célébration. Elle n'est donc pas soumise à un encadrement doctrinal, ni astreinte à une mission didactique et édifiante. Il se trouve que Marlowe possédait à la fois des connaissances théologiques et une intuition poétique suffisantes pour rendre émouvants les états de conscience du pécheur coupable de la transgression la plus grave. Mais ceci ne permet ni de supposer que Marlowe a vécu de semblables expériences ni de déceler 
ses intentions dans une pièce qui, comme la chronique dont elle est tirée, fait une large place à la farce et la fantasmagorie.

Deux données viennent d'ailleurs en compliquer l'examen. Il existe deux textes substantiellement différents et d'autre part il est très probable que l'oeuvre a été écrite en collaboration. Ne pouvant rouvrir le débat je renverrai à l'édition des deux textes par W.W. Greg, dont les conclusions sont assez généralement acceptées ${ }^{17}$. L'inquarto de 1604 (A) serait un texte reconstitué de mémoire tandis que celui de 1616 (B) serait fondé sur un manuscrit d'auteur ou un livre de régie (et secondairement sur A). A ne comportant que 1500 lignes, 600 de moins que B, serait une version abrégée, qui suppose un moindre déploiement d'effets scéniques. Les scènes omises en A concernent certains épisodes de la satire du Saint-Siège, certaines facéties du magicien, enfin la présentation, au dénouement, du Paradis et de l'Enfer (la seule qui touche directement au destin de Faust). On a supposé que ces scènes correspondaient à des adjonctions effectuées après la mort de Marlowe ${ }^{18}$. Mais des échos ou des allusions dans des pièces contemporaines semblent indiquer que l'une de ces scènes au moins est antérieure à cette date et que le texte $\mathrm{B}$ dans son ensemble n'est pas postérieur à 1594. Il est donc douteux que ces «additions» aient été incorporées aux textes imprimés.

Cependant ceci laisse entier le problème d'une collaboration car si la présence de Marlowe est sensible dans les scènes consacrées à la tragédie spirituelle de Faust on ne rencontre guère les marques ordinaires de son style dans les scènes comiques, et il est fort probable qu'une partie au moins soit d'une autre main. Mais la grandeur des scènes où se noue et se résout le destin de Faust ne doit pas inciter à considérer les autres comme secondaires et éventuellement détachables. On a vu qu'elles avaient une fonction dans un ensemble en même temps qu'elles répondaient à une demande du public. Et la pièce ne doit pas être jugée seulement comme une oeuvre d'un poète génial, mais sur son efficacité en tant que spectacle. Il est vraisemblable que Marlowe, même s'il a eu un ou deux collaborateurs, a été responsable de la conception d'ensemble, et de la division du travail. En présence de 
deux textes comportant des scènes en plus ou en moins, ou diversement rédigées, la solution idéale est l'édition de textes parallèles. Sinon il faut choisir A ou B, et après avoir effectué les émendations nécessaires, inclure dans l'appareil critique tout ce qui est en plus, en moins, ou différent dans l'autre texte. L'important est que le lecteur, y compris l'éventuel metteur en scène, puisse faire ces choix en connaissance de cause ${ }^{19}$.

Aussi grande que soit la part de la farce, de la satire, du merveilleux dans la pièce, le problème central de la tragédie de Faust reste celui du mécanisme de la damnation. D'entrée de jeu le héros est défini comme un savant qui refuse de croire à la possibilité du salut. C'est une donnée de base, de même que la cause de ce refus : son aveuglement à l'égard du pouvoir rédempteur du Christ. Ceci apparait dès le début dans le syllogisme par lequel il justifie son rejet de la théologie et qu'il construit à l'aide de fragments du Nouveau Testament pour conclure à la fatalité du péché et de la mort, alors que leur contexte immédiat proclame la purification par le sang du Christ ${ }^{20}$. Lecture pour le moins inattentive, qu'une intervention diabolique explique peutêtre (on y reviendra) mais qui résulte certainement de l'engouement qu'il manifeste aussitòt après pour la magie. Si Faust possède une parenté avec Tamerlan c'est ici qu'il faut la chercher. L'empire d'un bon magicien, dit-il, s'étend aussi loin que l'esprit humain et il veut s'efforcer par cet art de devenir divin (sc. I, 52-62). Ceci est un écho (affaibli il faut bien le reconnaitre) du célèbre monologue du conquérant (Our souls, Whose faculties cancomprehend The wondrous architecture of the world). "Sois sur la terre ce que Jupiter est dans les cieux» dit le Mauvais Ange qui exhorte Faust. Mais ici la ressemblance s'arrête. Tamerlan agit de sa seule volonté et toujours en dominateur, on ne l'imagine pas encadré de deux instances morales se disputant leur influence.

Le Bon et le Mauvais Ange sont des personnages scéniques extérieurs à Faust mais qui sont perçus par lui comme les voix d'un débat intérieur. Voix de la conscience et de la piété, voix des désirs interdits. A peine Faust a-t-il mentionné son projet d'être initié à la magie que le conseiller et le tentateur sont en alerte. Bientôt d'ailleurs 
il aura affaire à deux agents de la damnation, le Mauvais Ange, puis, lorsqu'il aura invoqué les esprits, Méphistophélès. Quant au véritable caractère de la magie qu'il va exercer, elle n'est révélée que progressivement, si bien que toutes les pratiques que ce nom recouvre paraissent également repréhensibles. Or on a vu qu'il existe une magie naturelle qui utilise uniquement les influences astrales et les propriétés physiques des corps, et que celle qui implique un commerce avec les esprits ne comporte pas de véritable risque si le magicien conserve sur eux son ascendant et son contrôle. Faust imagine, sur la foi de ses initiateurs, qu'il lui suffira d'invoquer les esprits pour en faire ses serviteurs, mais ceux-ci, le voyant entraîné par ses appétîts, n’auront pas de peine à imposer le pacte qui fera de lui leur esclave. En fait c'est tout un courant de la pensée de la Renaissance, nourri de l'apport hermétique et platonicien, qui se trouve incriminé dès les premières scènes, alors que les aspirations et l'esprit d'exploration qui l'animent se fondent sur la vision d'un univers aux correspondances innombrables, animé par le pouvoir divin.

Dans le monde du Dr. Faustus il n'y a pas de place pour d'autres esprits que ceux des hiérarchies célestes et ceux qui ont suivi Lucifer dans sa révolte et dans sa chute. D'ailleurs ce ne sont pas seulement les spéculations et les expériences de la magie qui sont déconsidérées, mais les mobiles de ceux qui s'y livrent. Non seulement Faust convoite les biens terrestres, pouvoir, richesses, honneurs, auxquels il sacrifiera sa part de vie éternelle, mais les avantages qu'il imagine ont un caractère à la fois démesuré et frivole, et ceux qu'il obtiendra seront en fin de compte dérisoires. Cependant les nobles aspirations ne sont pas absentes, en particulier la soif de connaissance, et ia perception de la beauté.

Il interroge Méphistophélès sur l'astronomie, mais celui-ci ne lui apprend rien sur l'architecture du monde qu'il ne sache déjà. Le sens de cette scène est de montrer que la contemplation de l'ordre de l'univers doit élever les pensées du savant vers son Créateur. Et c'est à la seule question : qui a créé le monde ? que le démon refuse de répondre (VI, 35-70). Le récit de l'exploration du cosmos par Faust monté sur un char magique (Chorus après la sc. VII, 1-14) est une image assez frappante de l'esprit 
d'observation, de même que le dialogue qu'on vient de citer illustrait l'exercice du raisonnement. Marlowe trouve aussi des accents harmonieux pour exprimer la consolation que son personnage demande à la poésie d'Homère et à la musique des Anciens (VI, 26-30). L'antiquité grecque est encore évoquée, avec les figures d'Alexandre et de sa maîtresse, puis d'Hélène. Tamerlan célébrait déjà la beauté pour laquelle mille vaisseaux avaient pris la mer, mais pour dire que la «divine Zénocrate» la surpassait encore (2 Tamb. II, iv, 85-90). Faust, lui, pense trouver le ciel sur les lèvres d'Hélène, mais il n'étreint que le succube qui a pris sa forme, et le baiser qui doit le rendre immortel est le baiser qui tue (XVIII, 99 et s.). La grande envolée lyrique est encore présente mais se charge d'ironie et d'ambiguïté, et l'image de la beauté est avilie.

Une étude récente a attiré justement l'attention sur la conduite de Méphistophélès, qui a pour mission d'attirer l'âme de Faust en Enfer mais qui témoigne à l'égard de sa victime une certaine compassion et l'informe charitablement de ce qu'elle ne devrait pas ignorer ${ }^{21}$. Cette attitude le rend plus intéressant qu'un agent d'exécution borné et permet d'exposer tout un aspect du dogme dans une pièce oủ les esprits du mal sont contraints de reconnaitre la loi divine. Faust n'est d'ailleurs pas une proie ordinaire. Il est mû par des aspirations qui rappellent au démon qui a répondu à son appel la révolte de Lucifer et la chute des anges qui l'ont suivi. Et les vers sur la souffrance d'être privé de la contemplation de la face de Dieu comptent parmi les plus mémorables du poète (III, 79-84). Cette scène montre aussi l'inconsistance du héros qui se croit déjà damné et disposé à livrer son âme en échange de quelques années de bonheur terrestre, et qui en même temps joue les esprits forts et nie l'existence de l'Enfer. Mais si le diable lui donne beaucoup de fil, il l'accroche sûrement à l'hameçon, sachant que Faust est prêt à tout renier pour obtenir ce qu'il désire. Il s'active pour lui faire rédiger le pacte et va chercher du feu (de l'Enfer) pour empécher que le sang du héros ne se fige. Episode d'une densité symbolique étonnante (l'acte contre nature, le sacrilège, la parodie du sacrifice du Christ).

Cependant malgré le pacte la grâce est toujours prête, comme le déclarent son Bon Ange, puis un saint Vieillard. 
Il suffit que Faust se repente, à condition que ce repentir soit complet et s'accompagne de la contrition et de la prière. Mais à l'absence d'espoir en la grâce viennent s'ajouter les obstacles de l'accoutumance et de l'endurcissement, et enfin les menaces et les tourments des démons, dès qu'il manifeste des signes de repentir. Lorsqu'il implore le Christ Lucifer intervient en personne et Faust terrifié promet de se soumettre et de se distinguer par son zèle satanique (VI, 85-103). La situation est renversée. Il s'adresse à Lucifer comme à son dieu et le supplie de pardonner ses offenses. Mais il suffit à ce dernier de le maintenir dans le divertissement et l'oubli du remords. C'est alors que se déroulent ses aventures à Rome et à la cour impériale, et diverses actions comiques auxquelles il se trouve mêlé. Et c'est seulement vers la fin que réapparaît le thème majeur.

Un Vieillard parvient à convaincre Faust que le repentir est encore possible. A et B donnent des versions assez différentes de ses paroles. L'intérêt du texte A en ce point est que les reproches y sont plus sévères et qu'il insiste sur la nécessité de la contrition (Breake heart, drop bloud, and mingle it with tears. Greg, A 1306). Méphistophélès, dans cette scène, se montre sous ses vraies couleurs. Il pousse Faust au suicide et menace de le déchirer dès qu'il tente de se libérer. Le schéma se répète et le héros, pour convaincre à nouveau son seigneur (Lucifer) de la sincérité de son repentir, demande au démon de tourmenter le saint Vieillard.

A partir de quel moment la grâce cesse-t-elle d'être possible ? Après ce nouvel échec Faust fait part à ses anciens compagnons d'étude de la servitude dans laquelle il s'est mis. Il désespère toujours, et le diable paralyse ses efforts : Ah, my God, I would weep, but the devil draws my tears ... (XIX, 56 et s.) et cette situation se reproduira à son heure dernière lorsqu'il verra ruisseler au firmament le sang du Christ dont une seule goutte suffirait à le racheter, puis se substituer à cette image le visage d'un Dieu de colère. On sait alors que la grâce a été refusée. Mais B comporte des fragments importants qui manquent en $\mathrm{A}$ et qui, sans modifier le dénouement, font voir sous un jour différent le processus de la damnation.

Lorsque ses visiteurs l'ont quitté, Méphistophélès apparaît à Faust et lui révèle que c'est lui qui l'a tenté et 
mis sur le chemin de la damnation, plus précisément que c'est lui qui a tourné les pages et conduit ses yeux lorsqu'il a consulté les Ecritures (XIX, 92-6). Ceci correspond exactement à ce qui s'est passé dans la première scène au moment décisif ou Faust, feuilletant la Bible, a vu ce qui concernait le péché et la mort, et non le promesse du salut. Le démon serait-il donc la cause première de la damnation ? non car il ne peut faire que ce que Dieu lui permet, mais incontestablement il est une agent de la damnation.

L'irréversibilité du châtiment de Faust est mis en évidence, aussitôt après, avec l'apparition du Bon et du Mauvais Ange qui lui montrent respectivement, d'une manière crûment réaliste, les joies du Ciel qu'il a perdues et les tourments de l'Enfer. Dans cette scène la damnation est acquise au moment où, après l'avoir morigéné, le Bon Ange abandonne Faust, le livrant en quelque sorte au bras séculier. Cette scène a été rejetée comme grossière et indigne d'exprimer le message évangélique de la pièce: qu'il faut préférer à tous les biens du monde le salut de son âme ${ }^{22}$. Mais nous voyons pourtant, dans le monologue final commun aux deux versions, se renforcer les uns par les autres et se confondre les tourments physiques et spirituels de Faust. Son dernier discourss illustre la désintégration d'un homme terrifié. Il est fait de supplications vaines et de souhaits impossibles, mais garde cependant une certaine cohérence argumentative : si la grâce est refusée, que du moins je puisse me dissoudre et échapper à la colère divine, ou bien que mon supplice, aussi long soit-il, ait une limite. Mais c'est en vain que le savant qui a compris l'architecture de l'univers implore les corps célestes d'interrompre leurs mouvements, et les éléments de le cacher ou de le détruire. Ses dernières paroles se réduisent à des cris, et débouchent sur la confusion d'un Enfer où tout est converti en souffrance absolue : My God, my God! Look. not so fierce on me! Adders and serpents, let me breathe a while! Ugly hell, gape not Come not, Lucifer: I'll burn my books ... Semblable éclatement de la pensée et du langage se recontrait déjà dans les exclamations de l'épouse de Bajazet au bord du suicide. Et force est de constater l'intérêt particulier de Marlowe pour les aspects répressifs du dogme, pour les grands rôles de rebelles et de bourreaux, Tamerlan et Lucifer, et d'une manière plus générale pour les rapports 
de persécuteur à persécuté, pour la souffrance infligée ou subie.

Les dernières paroles de Faust sont pour abjurer son savoir. Et certes l'on peut supposer que les livres qu'il voue au feu sont ceux dont it s'est servi pour invoquer les démons. Mais prenant congé de ses compagnons il attribuait sa damnation à ses études $(O$, would $I$ had never seen Wittenberg, never read a book) et le Chorus final met en garde ceux qui voudraient pénétrer des secrets trop profonds et entreprendre plus qu'il n'est permis.

Faust est une tragédie de la connaissance mais son protagoniste, plutôt qu'un héros prométhéen, est un personnage non sans grandeur mais assez faible, que ses errements vouent à la défaite. La frontière entre licite et interdit est définie en termes de théologie et de savoir traditionnel. Au delà s'étend le domaine de la «magie» qui est assimilée tout entière au commerce avec le diable alors que ce mot recouvre tout un courant de spéculation et d'exploration.

Ce qui est en jeu c'est aussi l'extension du pouvoir sur la nature, la valorisation de la vie terrestre avec pour contrepartie le sens de la fuite du temps et le sentiment de la mort. Mais le monde de profit et de plaisir que Faust désire se révèle illusoire. Dans la pièce il apparaît vaincu d'avance, étant certain d'être damné. La récurrence de la grâce offerte et du repentir inabouti est dramatiquement forte, mais il est difficile de dire jusqu'à quel moment cette gráce est encore possible, ou même si elle l'a jamais été. D'où l'impression d'une liberté qui, vue d'un autre angle, apparait comme une fatalité qui réduit le protagoniste au rôle d'une créature prise au piège.

Dr. Faustus est une étape décisive dans la formation d'un des grands mythes de l'Occident. L'ensemble de ses deux versions permet d'y mesurer l'apport de la tradition médiévale, dans ses aspects populaires et doctrinaux, et d'y discerner aussi les préoccupations de la conscience réformée, et une interrogation sur les voies de la justice divine. L'appareil répressif est montré sous son jour grotesque et terrible. Mais l'originalité de Marlowe est sans doute d'avoir fait du damné une figure tragique, invitant les spectateurs, à la dernière scène, à concentrer leur attention sur sa solitude et sa souffrance, plutôt qu'à jubiler devant 
l'exécution de la vengeance du ciel.

P.S.

C'est le contraire qui se passe pour Barabas bien que selon les interprétations typologiques qui ont été proposées ils représentent l'un et l'autre l'inversion d'un modèle chrétien, Faust suivant la carrière d'un saint à rebcurs, Barabas celle d'un serviteur de Mammon. Mais bien que divers schémas théologiques y soient discernables, Le Juif de Malte s'organise selon la logique d'une tragédie de la vengeance, utilisant les ressorts de la comédie noire, et combinant le grotesque et l'ironie. Marlowe y développe une technique déjà expérimentée dans les scénes de la violation du serment dans Tamburlaine. Il fait ressortir l'écart entre les professions de foi et les actes, et la relativité des points de vue religieux. Les juifs sont vus par les chrétiens, et inversement, et en principe sa critique neépargne ni les uns ni les autres.

Côté chrétien ce n'est pas seulement le «papisme» qui, par le biais de la satire des moines, est mis en cause. C'est l'indignité de ceux qui, comme les Chevaliers de Jésusalem, devraient constituer l'élite de la chrétienté. Le Gouverneur de Malte rançonne la communauté juive pour payer le tribut au Sultan, vend les musulmans comme esclaves et détruit l'armée turque grâce à la ruse de Barabas, conduite qui donne sa saveur caustique au mot de la fin (let due praise be given Neither to fate nor fortune, but to heaven).

Cependant on chercherait en vain un plaidoyer cohérent contre les préjugés de religion. Le «machiavélisme» de Barabas, mis au service de l'appétît des richesses, est défini dès le prologue comme amour de soi absolu et négation de toute loi morale. Présenté sans indulgence mais avec une relative objectivité dans les fortes scènes du début il est appelé par la suite à jouer le rôle d'un «villain», d'une figure démoniaque vouée finalement à un rituel d'élimination. C'est ainsi qu'il acquiert cette licence de parole, propre à un maître-intrigant cynique, qui s'exercera avec verve jusqu'au moment où il succombera à son proprc stratagème. Si bien que le spectateur est invité à juger de la duplicité politique et de la cupidité des chrétiens, qu'il perce à jour, en le prenant pour référence. Le pire reproche 
qu'on puisse leur faire c'est d'être tels que Barabas. Et ce jeu d'un miroir déformant à l'intérieur de la pièce la rend difficile à porter à la scène aujourd'hui.

\section{Jean JACQUOT}

\section{NOTES}

1. La place manque pour une esquisse de l'état des études marloviennes, ou même pour une bibliographie sélective. Il va de soi que les travaux qui ont fait progresser ces études ne se ramènent pas tous à l'une ou l'autre de ces tendances.

2. Seule la première partie a puétre présentée à la Table ronde. La seconde, dans le texte que voici, a été limitée, pour des raisons d'espace, à Tamburlaine et a Dr. Faustus, une discussion plus détaillée de ces deux oeuvres ayant été jugée préférable à un survol de l'ensemble. J'ai tenu compte, dans cette rédaction finale, des questions soulevées dans les communications de Mme Sybil Truciet, de Professor Roma Gill et de M. François Laroque ainsi que des échanges de vues entre participants lors de cette Table ronde, et au cours d'une séance préparatoire qui avait réuni la plupart d'entre eux.

3. Enquête menée à Cerne, Dorsetshire, en mars 1594 Documents publiés par F.C. DANCHIN dans «Etudes critiques sur C. Marlowen, Revue germanique, 1913, pp. 578-87.

Discussion dans Pierre LEFRANC, Sir Walter Ralegh écrivain l'oeuvre et les idées, Paris 1968, pp. 379.93.

4. $\$$... or have sayde those Scriptures are not to be helieved and defended by Her Maiestie for doctrine and faith and salvacion. but onlye of policye, or civell governmenty. DANCHIN, ibid. p. 579.

5. DANCHIN, ibid., pp. 568-78 (voir également F.S. BOAS, C. Marlowe, a biographical and critical study. Oxford 1960); LEFRANC, ouv, cit., pp. 361-379 et App. L)

6. Sur la réputation de $R$ alegh, sa pensée philosophique et religieuse voir LEFRANC, ibid., ch. XII-XIII.

7. Spaccio III, ii, Opere italiane, ed. $G$. Gentile, Bari 1927. vol. 2. p. 178. - Dans son livre magistral. Giordano Bruno and the Hermetic tradition, Londres 1964 . Frances A. YATtS met bien en évidence la prépondérence de cet apport hermétique dans l'oeuvre de Bruno. convaincu de la supériorité de la tradition égyptienne sur la tradition juive

8. Stimulés par lorganisation de séminaires à All Souls College, Oxford, à l'université de Durham, et à l'université de 
Defaware.

9. On trouvera ma contribution sur ces points dans $T$ Thomas Harriot's reputation for impiety», Nores and Records of the Royal Society of London (IX) 1952, pp 164.87 et dans wHarriot, Hill, Warner and the New Philosophy, Thomas Harriot Renaissance Scientist, ed. John SHIKLEY, Oxford 1974, pp. $107-28$.

10. Dr. Faustus ayant été vraisemblablement composée après Ia publication en version anglaise de la source principale, The History (...) of Doctor John Faustus, en 1592 (probablem ent en mai).Voir l'édition de la pièce par John D. JUMP, Revels Plays, London 1962. pp x xiv-xxv

11. 1, II, vii, 12-29. Ed, utilisée: U.M. ELLIS-FERMOR, London 1930. -. Dans un article ancien ( $L$ a pensée de Marlowe dans Tamburlainc the Great, Etudes anglaises, 1953, 332-45) j'a i proposé une interprétation de quelques monologues de Tamerlan qui me paraît encore défendable. Mais je ne tenterais plus a jourd'hui de suivre la progression de la pensée de Marlowe à travers cette we uvre.

12. Une taille élancée comme ses désirs, un cielétollé dans les regards, $m$ ais aussi le charme ambigu des boucles caressées par la brise (1. $11, i, 8$ et s.)

13. Leur assimilation. par Mme $S$. Truchet, au Roi et à la Reine du Grand Oeuvre, est révélatrice et met bien en évidence limportance du symbolisme dans cette première partie. Mais jy verrais une composante, ou un niveau d'interprétation. plutót qu'un theme centrat.

14. "J'aimais jusqu'à ses pleurs que je faisais couler $\# .$. . Brûlé de plus de feux que je n'en allumain (... Lenocrate; whose sorrows lay more siege unto my soul Than all my army to Damascus' walls.)

15. 2, IV, i, 146-58. Voir mon commentaire dans l'art. cité n. 11, p. 336 .

16. Cif. 2, IV, i, $178-81$ et 2, V, iii, 82 ets.

17. Marlowe's Doctor Faustus, 1604-1616. Parallel tex/s. Oxford 1950. Voir aussi lintroduction de John D. JUMP à son édition, citée plus haut n. 10. 1602 .

18. "Additions\% payées à Birde et Rowley par Henslowe en

19. La reconstitution d'un texte unique de Dr. Faustus, comme celle qu'a proposée W.W. GREG en marge de son èdition parallèle, dans $A$ Conjectural reconstruction, Oxford 1950 , implique inévitablement des choix personnels, et dépasse les limites d'une édition de texte.

J'ai utilisé pour cette étude les textes parallèles de fireg et 
l'édition Jump. Elle est fondée sur B mais répond aux critères que je viens de définir. - Dans la communication publiée dans ce volume Professor Roma Gill expose, avec sa longue expérience et sa sensibilité au texte de Marlowe, ses préférences pour le texte A.

20. Sc. 1, 37-47, Cf. Rom, V1, 23 et 1 Jean 1, 8-9.

21. Claudine DEFAYE, «Mephostophilis est-il un démon authentique ?\%, Etudes anglaises, 1979, pp, 1-10.

22. C'est la position de Professor Roma Gill dans sa communication 